\title{
Integrated Palliative Care Outcome Scale (iPOS) as a Tool to Identify Complex Palliative Care Needs - First Results of iPOS-assessment in a Palliative Care Team
}

\author{
Roch C, Noeske L, Oorschot B.
}

Interdisciplinary Centre Palliative Care, Department of Radiation Oncology, University Hospital Wuerzburg, Germany.

Background: All patients who are considered by professionals to profit by palliative Care services our in-house palliative care service (PCS) will be consulted. At the beginning of the medical support service the iPOS will be performed. The iPOS questionnaire can be fulfilled either by patients or by professionals and includes three dimensions: physically, emotionally and practicably.

Aim: We expected that the need for palliative care service could be figured out by observing the iPOS summary scale as well as by observing the single items.

Methods: We retrospectively collected the iPOS scores and single items from in-house patients (01.01.2018 until 29.10.2018) from electronically patients records, who were presented to the palliative care service. Two groups were built, those who were supported by the palliative care service and those who were either advised by the PCS or not even supported. A t-test on the summary score and the single items was performed.

Results: 747 (388 male/ 356 female/ 3 not mentioned) patients were presented to the PCS. 209 patients were supported by the PCS, 371 were either advised or not supported. Oncologically diseased were 558 patients, not oncologically diseased were 112 patients and 77 patients illnesses were not mentioned. At all we received 303 complete datasets, of which 272 were oncologically and 26 not oncologically diseased, 5 missing information of the underlying disease. Considering the summary score we figured out, that the summary scores of supported patients were not significant $(p=0,146)$ higher than those who were not supported and/or advised. If the single items, concerning the severity of symptoms, were considered, there is no significant difference between physical symptoms in supported or not supported patients.

\begin{tabular}{|l|l|}
\hline Total Number & 747 \\
\hline Complete questionnaires & 303 \\
\hline Male/ female/ no naming & $135 / 167 / 1$ \\
\hline Median/ Mean (Range years) & $67 / 66,7$ (31-95) \\
\hline Primary disease & \\
\hline Oncologically diseased & 272 \\
\hline not oncologically diseased & 26 \\
\hline Missing data & 5 \\
\hline Supported by PCS & 172 \\
\hline not supported or advised & 131 \\
\hline
\end{tabular}

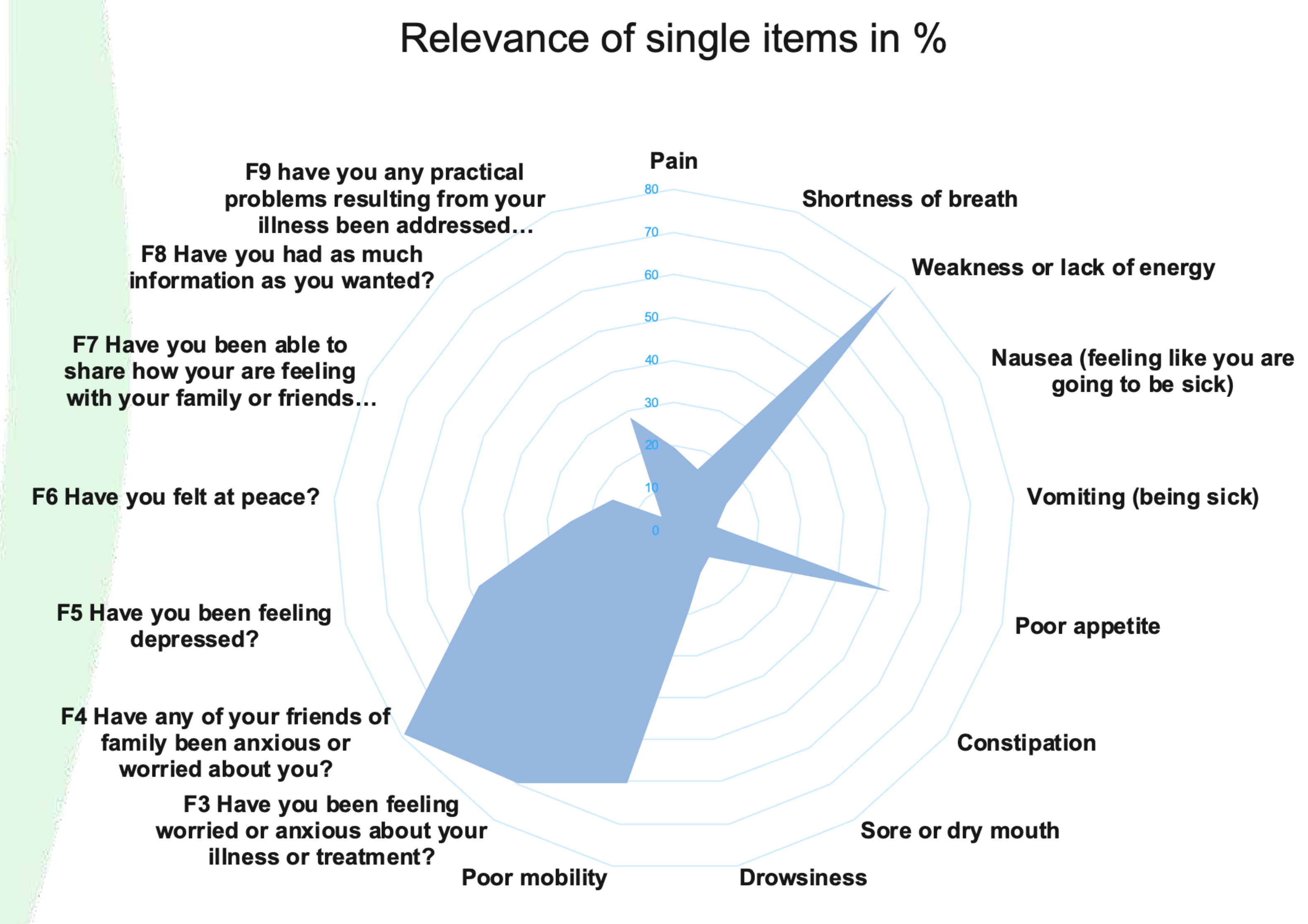

Conclusions: Assigning patients to those who receive palliative care and those who don't, can't reliably be predicted by using measurement tools like iPOS. Also the single items cannot be considered on their own. Nevertheless the emotional or practical dimension seems often to be as important as the physical dimension. 